\title{
Adenolymphoma of the lacrimal gland: A very rare case report
}

\author{
Deepanjan Ghosh ${ }^{1}$, Jayant Ekka ${ }^{2, *}$ \\ ${ }^{1}$ Registrar, ${ }^{2}$ Post Graduate Student, ${ }^{1,2}$ Dept. of Ophthalmology, Assam Medical College, Dibrugarh, Assam, India
}

*Corresponding Author:

Email: ekkajayant@gmail.com

\begin{abstract}
Adenolympoma also known as warthin's tumor is the second most common benign tumor of the parotid gland, characterized by slowly growing painless mass occurring in elderly people over the age of 60 years. Histopathologically, it is characterized by multiple cystic spaces lined by double layered columnar epithelium interspersed with the lymphoid tissue. Adenolymphoma of the lacrimal gland is very rare in clinical practice. We are presenting a case of 45 year female who presented with a painless mass in the upper outer part of the left eye of two months duration. CT orbit revealed a homogenous soft tissue mass in the superolateral aspect of left orbit with possibility of lymphoma. USG guided FNAC was done and showed mixed cellularity with small to intermediate lymphoid cells. A lateral orbitotomy was performed and mass was excised completely and sent for histopathological evaluation. Histopathological section showed small groups of normal looking glandular elements surrounded by lymphoid tissue. No evidence of malignancy seen. Picture suggestive of Adenolymphoma of salivary origin.
\end{abstract}

Keywords: Adenolymphoma, Warthin's tumor, Lacrimal gland.

\section{Introduction}

Adenolympoma also known as warthin's tumor is the second most common benign tumor of the parotid gland after pleomorphic adenoma. Although the tumor occasionally occurs in the periparotid lymph nodes, it is rare in the other salivary glands. Warthin tumor is associated with cigarette smoking and ionizing radiation and is traditionally described as a tumor of elderly males. The tumor classically presents as a slowly enlarging painless mass, although a minority demonstrates rapid enlargement with associated pain or pressure symptoms. Malignant transformation is very rare.

On ultrasonography a well-defined hypoechoic mass is characteristic, often containing multiple small cystic areas, but occasionally an almost entirely cystic mass is seen. Warthin's tumor is an adenoma composed of bilayered columnar and basaloid oncocytic epithelium that forms multiple cysts with numerous papillae, accompanied by a proliferation of folliclecontaining lymphoid tissue.

Occurence of this tumor in the lacrimal gland is very rare with only one case reported in the literature till now. ${ }^{1}$

\section{Case Report}

A 45 years old female patient presented with a mass in the upper outer part of the left orbit since 2 months. (Fig. 1)

Onset was insidious with gradual progression without pain causing gradual forward bulging of the left eye. There was no history of trauma, diminution of vision, pain, redness, restriction of movement, watering and diplopia. There was no other significant surgical or medical history.

On examination the BCVA was 6/6, N6 for both eye. There was mass in the supero-temporal region of the left orbit, firm in consistency. Skin over the mass was freely mobile. There was no tenderness and erythma.

Transillumination was negative. There was proptosis of $28 \mathrm{~mm}$ noted in anterior-posterior direction in the left eye. (Figure 2) Extraocular movement was normal in all cardinal gazes. There was no lid lag and/or lid retraction. Bell's phenomenon was normal. Anterior and posterior segment of both eyes were normal.

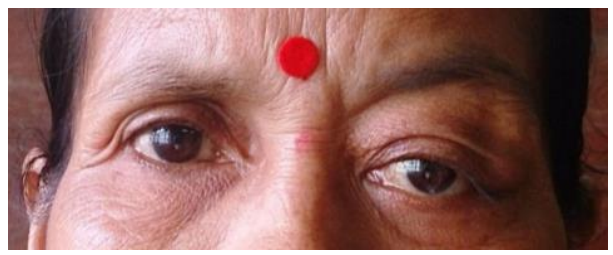

Fig. 1: Mass in the upper outer part of the left orbit

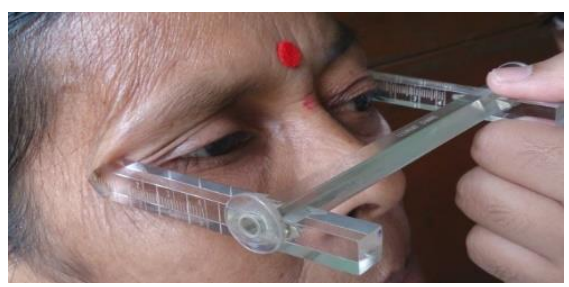

Figure 2: Measurement of proptosis

Routine blood investigations was normal. Chest Xray revealed normal study. NECT orbit revealed an extraconal homogenous soft tissue density lesion in supero lateral aspect of left orbit in the region of the lacrimal fossa suggestive of Lymphoma. (Fig. 3)

FNAC showed mixed population of small to medium lymphoid cells with irregular nuclei, with mature lyphocytes in hemorrhagic background. 
A lateral orbitotomy was done and the mass was fully excised and the specimen was sent for histopathological examination.

Histopathological section showed small groups of normal looking glandular elements surrounded by lymphoid tissue. No evidence of malignancy was seen. Picture was suggestive of Adenolymphoma of salivary origin. (Fig. 4)

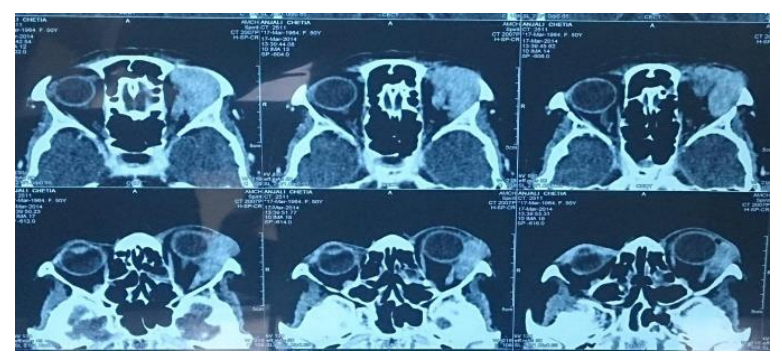

Fig. 3: NECT orbit showing an extraconal homogenous soft tissue density lesion in supero lateral aspect of left orbit in the region of the lacrimal fossa

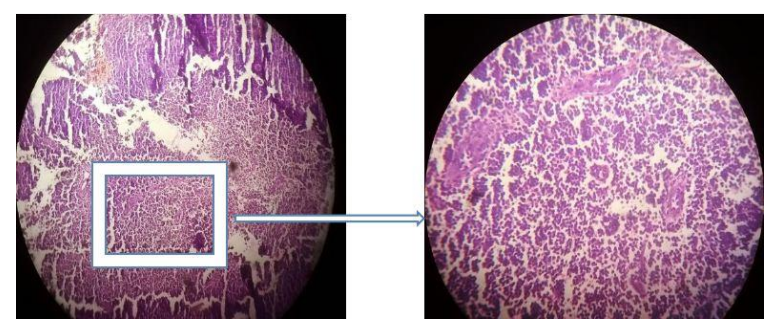

Fig. 4: Histopathological section showing small groups of normal looking glandular elements surrounded by lymphoid tissue

On the basis of history, clinical features on examination, findings on radiological investigations and histopathological evaluation the diagnosis of Adenolymphoma of the Lacrimal Gland was made.

\section{Discussion}

Adenolymphoma of lacrimal gland is of very rare occurrence. The first reported case was by Bonavolonta G et al. ${ }^{1}$
Tumors of the lacrimal glands are similar to those arising in the salivary glands except for Warthin tumor, which is common in the major salivary glands but is virtually never found in the lacrimal glands, with only one well-documented case reported to date. ${ }^{1}$

Warthin's tumor is thought to be a disease of male. Our patient was a female of 45 years. In a study it was found that the mean age of presentation of benign lacrimal gland tumors is 41-47 years. ${ }^{2}$

In a study done by M.kohli et al they mentioned that benign tumors present with a slowly progressive painless mass at the supero temporal region. ${ }^{3}$

Our patient also presented with slowly growing painless mass in the supero-temporal region of the orbit.

Complete excision of the mass is the treatment of choice in all cases of benign tumors of the lacrimal gland.

\section{Conclusion}

Adenolymphoma of the lacrimal gland is very rare occurence. Benign tumors of the lacrimal gland present as painless mass involving the supeo-temporal aspect of the orbit with resulting displacement of the eyeball. Complete excision of the mass is the treatment of choice in all cases of benign tumors of the lacrimal gland.

Funding: No funding sources.

Conflict of interest: None declared.

\section{References}

1. Bonavolontà G1, Tranfa F, Staibano S, Di Matteo G, Orabona P, De Rosa G : Warthin tumor of the lacrimal gland. Am J Ophthalmol 1997;124(6):857-58.

2. N Chuo, K Ping-Kuan, TP Dryja : Histopathological classification of 272 primary epithelial tumors of the lacrimal gland. Chinese Med J 1992; 6:481-85.

3. Kohli M, Shah A, Bhatt S, Aggarwal S : Lacrimal Gland Tumors - A Retrospective Histopathological Study. Gujrat Med J 2011;66(1).

How to cite the article: Ghosh D., Ekka J. Adenolymphoma of the lacrimal gland: A very rare case report. Int $J$ Ocul Oncol Oculoplasty 2018;4(3):144-145. 